\title{
The Association Between Healthcare Resources, Non- communicable Diseases, and Covid-19 Mortality: An Epidemiological Study of 139 Countries
}

\author{
Shahram Arsang-Jang \\ Zanjan University of Medical Sciences \\ Masoud Tokazebani Belasi \\ Iran University of Medical Sciences \\ Farid Najafi \\ Kermanshah University of Medical Sciences \\ Mitra Darbandi \\ Kermanshah University of Medical Sciences \\ Malik Zain Raza \\ Western University \\ Humayon Akhuanzada \\ University of Melbourne \\ Nawaf Yassi \\ University of Melbourne \\ Jose Biller \\ Loyola University Health System, Stritch School of Medicine \\ Ramin Zand \\ Geisinger Medical Center \\ Sepideh Kazemi Neya \\ Iran University of Medical Sciences \\ Negar Morovatdar \\ Mashhad University of Medical Sciences \\ Saverio Stranges \\ Western University \\ Mario Di. Napoli \\ San Camillo de' Lellis General District Hospital \\ M. Reza Azarpazhooh ( $\sim$ reza.azarpazhooh@lhsc.on.ca ) \\ Western University
}

\section{Research Article}

Keywords: COVID-19, health infrastructure, healthcare resources, the burden of disease, mortality, health access and quality index, health expenditure

Posted Date: April 22nd, 2021 
DOI: https://doi.org/10.21203/rs.3.rs-379944/v1

License: (c) (i) This work is licensed under a Creative Commons Attribution 4.0 International License. Read Full License 


\section{Abstract}

Background: To provide an overview of the worldwide association between hospital beds, the burden of noncommunicable diseases (NCDs), and COVID-19 mortality.

Methods: Data was extracted regarding COVID-19 deaths and cases from the Our World in Data as of March 23, 2021. The following data was obtained:1) NCDs disability-adjusted life years (DALYs), health-adjusted life expectancy, and the health access and quality index from the Global Burden of Disease study; 2 ) the number of hospital beds, physicians, nurses and midwives per population, and out-of-pocket payments from the WHO website. Using the multilevel generalized linear model, these variables' independent associations with COVID-19 mortality rate ratio (MRR) was examined.

Results: Hospital beds were associated with reduced COVID-19 mortality (MRR=0.47; $95 \%$ Cl: 0.44 to 0.5 ) globally. During COVID-19 peak periods, despite a decreasing trend in COVID-19 MRR with increasing beds in high-income countries, the odds of mortality remained high even within the highest percentile of hospital beds (MRR=1.54 for $20^{\text {th }}$

$40^{\text {th }}$ and 1.06 for $>60^{\text {th }}$ bed percentile, respectively). On the contrary, in middle-income countries, an inverse association was observed between the number of hospital beds and COVID-19 mortality in both periods. NCD DALYs were associated with increased COVID-19 deaths, particularly during peak mortality periods in high-income countries. Death-to-case ratio increased by approximately two times during the peak vs non-peak mortality periods.

Conclusions: COVID-19 is a syndemic interacting with non-communicable diseases and not only a pandemic. A comprehensive national healthcare plan against COVID-19 spread should include adequate measures to protect vulnerable patients with pre-existing chronic conditions.

\section{Background}

A preventive model against COVID-19 should account for non-communicable diseases (NCDs). We have previously shown a significant correlation between NCDs and confirmed COVID-19 cases and deaths. ${ }^{[1,2]}$ Specifically, the burden of all NCDs measured by disability-adjusted life years (DALYs) was correlated with COVID-19 cases and deaths. ${ }^{[1,2]}$ Ischemic heart disease, ischemic stroke, and particularly dementia DALYs ${ }^{[1]}$ were correlated with COVID-19 deaths. ${ }^{2}$ These two studies indicated a higher number of COVID-19 deaths in high-income countries, which can be potentially explained by the ageing populations. ${ }^{[1,2]}$

Despite overall better healthcare in many High-income countries, we have seen a higher number of cases and deaths in these countries compared to low-income countries and middle-income countries. Although underreporting in lowand middle-income countries could be a plausible explanation, several other alternative hypotheses such as differences in population age and density, chronic disease prevalence, and healthcare resources require further investigation.

In the current study, we aimed to provide information regarding healthcare resources and the burden of noncommunicable diseases (NCD-DALYs). We also assessed correlations between healthcare resources, particularly hospital beds and COVID-19 mortality.

\section{Methods}

\section{Databases}


Using the Our World in Data database, we extracted data regarding COVID-19 cases and deaths per 1,000,000 population as of March 23, 2021. Briefly, the Our World in Data database provides up-to-date COVID-19 data according to the Centre for System Science and Engineering (CSSE) at John Hopkins University. ${ }^{[3]}$ Using the global burden of disease study (GBD), we obtained data regarding NCD DALYs (per 100,000 with 95\% uncertainty intervals [UIs], from GBD 2019), Healthy life expectancy (HALE, from GBD 2019), and the health access and quality index (HAQI, from GBD 2016). ${ }^{[4,5]}$ We used the World Health Organization (WHO) database to extract information about healthcare resources, including the number of hospital beds (per 10,000 populations), the number of physicians, nurses, and midwives (per 100,000 population), and out-of-pocket expenditure as a percentage of total expenditure on

health. ${ }^{[6]}$ Data regarding the gross domestic product (GDP) per capita was obtained from the Our World in Data database. Using the World Bank database, we extracted data regarding population density (people per $\mathrm{km}^{2}$ of land area), and income classification of countries into Lower-income countries, lower- Middle-income countries, upperMiddle-income countries and High-income countries. ${ }^{[7]}$

\section{Statistical analysis}

We used Spearman correlation to assess the association between the study variables and COVID-19 mortality. Multicollinearity was measured using a generalized variance inflation factor. Values with a factor $>2$ were considered a collinearity. We used a multilevel generalized linear model to identify the associations between study variables and COVID-19 mortality rate ratio (MRR). Country (as a variable) and income levels were included as a random effect in the model. Healthcare resources (i.e. number of hospital beds, physicians, nurses and midwives, and HAQI), HALE, NCD DALYs, GDP per capita, out-of-pocket payments, median age, and population density were included as fixed effects in the model. Due to over-dispersion in COVID-19 mortality rate, we used a negative binomials link function in the multilevel model. To address any multicollinearity problems, we used a principal component analysis. Hospital beds had a positively skewed distribution pattern. Accordingly, this was entered into our analytic model in quintiles (or combination of quantiles), excluding Lower-income countries due to inadequate sample size. COVID-19 peak mortality periods ( $\geq$ percentile 75th of COVID-19 death rate) were identified for each country. We then compared the association between hospital beds per quantiles and COVID-19 mortality rate during peak and non-peak periods. We also assessed the association of NCD DALYs (categorized according to their median values) with COVID-19 mortality during peak vs non-peak mortality periods. We used the Poisson-Tweedie mixed-effects model to track the risk of the death-to-case ratio and compare it between non-peak and peak mortality periods. Death-to-case ratio was calculated based on total number of deaths and cases in each country. To track changes in this ratio, we also calculated its difference by subtracting the ratio obtained during non-peak mortality periods from the ratio calculated during peak mortality periods. We used hierarchical cluster analysis to compare clusters of countries according to their death-tocase ratio during non-peak and peak mortality periods.

We used, plyr, pls, dendextend, and ggplot2 packages to implement analysis in the software R 4.03. A p-value $<0.05$ was considered statistically significant.

Data sharing: All data is publicly available with no individual identifiers.

\section{Results}

In the current paper, we obtained the following data: 1) COVID-19 cases and deaths (Supplemental Table 1); 2) Total country population, median age, senior citizens (aged>65 and aged>70 years) per population, and life expectancy (Supplemental Table 2); 3) Healthcare resources including the number of beds, physicians, nurses and midwives, GDP per capita, and out-of-pocket spending (Supplemental Table 3); 4) NCD-DALYs, HALE, and access to healthcare (HAQI) 
(Supplemental Table 4). The final analysis included data from 139 countries which had all the information needed for our study.

\section{COVID-19 mortality, healthcare resources, and ageing}

As of March 23, 2021, 35.69 COVID-19 deaths per 100,000 population have been reported globally, including 2.5 (95\%Cl: $2.5,2.502)$ in low-, 11.2 (95\%Cl: 11.204, 11.205) in lower-Middle-income countries-, 35.46\% (95\% Cl: 35.461, $35.462)$ in upper-middle-income countries, and 112.47 (95\% Cl: 112.47, 112.48) in High-income countries. Correlations between variables and possible collinearity are summarized in Supplemental Figure 1.

High-income countries had a higher median number of all healthcare resources as compared to lower-middle-income countries $(p<0.0001)$ (Supplemental Figure 2). A positive independent association was observed between COVID-19 mortality rate and median age ratio (MRR=1.077 [1.07, 1.08]).

\section{COVID-19 mortality, pandemic phases, and number of hospital beds}

In the multilevel model, lower COVID-19 mortality was associated with higher numbers of hospital beds, with the greatest reduction in COVID-19 deaths seen in the highest percentile of hospital beds (for $>$ Percentile $80^{\text {th }}$ MRR: 0.53 $[0.49,0.56]$, Table 1).

In a subgroup analysis based on COVID-19 mortality periods, the number of hospital beds per population reduced COVID-19 mortality in both peak and non-peak mortality periods (Figure 1-A). A similar pattern with an inverse association between COVID-19 MRR and the number of hospital beds was observed in both COVID-19 peak and nonpeak mortality periods in middle-income countries (Figure 1-B).

In a subgroup analysis, in lower-middle-income countries, we observed a declining trend of COVID-19 mortality with an increasing number of hospital beds during both peak and non-peak mortality periods. In upper-middle-income countries, we found the following declining trend only during non-peak mortality periods (COVID-19 MRR for hospital beds per population $<20^{\text {th }}$ percentile: $0.80[0.72,0.87] ; 20^{\text {th }}-40^{\text {th }}$ percentile: $0.68[0.60,0.77]$; and $>60^{\text {th }}$ percentile: $0.56[0.50,0.63])$. However, during peak mortality periods a non-significant increase in COVID-19 mortality was observed in upper-middle-income countries with an increasing number of hospital beds (COVID-19 MRR for hospital beds per population $<20^{\text {th }}$ percentile: $0.77[0.68,0.86]$; $20^{\text {th }}$ to $40^{\text {th }}$ percentile: $1.05[0.91,1.22]$; and $>60^{\text {th }}$ percentile: $0.90[0.79,1.02])$.

In High-income countries during peak mortality periods, despite a declining trend in COVID-19 mortality rate with an increasing number of hospital beds, the odds of COVID-19 mortality increased even with beds per population above 60th or 80th percentile (COVID-19 MRR for hospital beds per population 20th-40th percentile: 2.19[2.39, 3.74]; >60th: 2.59[1.17, 1.94]; >80th: $2.50[1.27,1.68]$ ) ( Figure 1-C).

\section{The association between the number of COVID-19 cases and deaths}

During the period of study, 124,535,520 confirmed COVID-19 cases were reported globally. Globally the death-to-case ratio was $2.2 \%$, ranging from $1.85 \%$ to $2.56 \%$ during non-peak and peak mortality periods, respectively (Table 2 ). In the Poisson-Tweedie mixed-effects model, we observed a significant interaction effect of "COVID-19 cases $\times$ periods" on COVID-19 mortality rate (interaction effect: 3.79 [3.62, 3.97]). Globally, the risk of death-to-case ratio increased significantly by 1.95 times $(1.75,2.18)$ during peak mortality periods compared to the non-peak periods, with a different pattern in countries depending on income levels (Lower-middle-income countries: 1.3[0.99, 1.71]; upper- 
middle-income countries: 1.77 [1.43, 2.20]; High-income countries: $2.14[1.79,2.57])$. We were not able to analyze lower-income countries due to missing information.

\section{Death-to-case ratio during non-peak and peak mortality periods: ranking and clustering of 139 countries}

We categorized all countries according to the death-to-case ratio in non-peak and peak mortality periods. Death-tocase ratio during peak mortality periods compared to non-peak mortality periods increased significantly in 121 countries. The greatest changes can be seen in Bhuran and Yemen followed by NewZealand as a High-income country (Supplemental Figure 3). In 10 countries (Albania, Colombia, Brazil, Hungary, Bangladesh, Morocco, India, Macedonia, Egypt, and Saudi Arabia) the increase in death-to-case ratio did not reach significance. The death-to-case ratio decreased during peak mortality periods only in eight countries (Algeria, Azerbaijan, Belarus, Serbia, Moldova, Turkey, Indonesia, and Romania). In the hierarchical clustering analysis of countries, we observed a significant change in the clustering of countries according to their death-to-case ratio during the peak mortality periods compared to non-peak periods (Supplemental Figure 4).

\section{COVID-19 and the burden of non-communicable diseases}

The burden of NCDs was associated with increased COVID-19 mortality rate globally in both peak and non-peak mortality periods (Figure 2-A). This association was, in particular, more evident in High-income countries where NCD DALYs increased COVID-19 mortality rate by $1.45(1.35,1.56)$ and $3.24(3.04,3.44)$ during non-peak and peak mortality periods, respectively (Figure 2-C). In upper-middle-income countries, during non-peak and peak mortality periods, NCD DALYs increased COVID-19 mortality by $0.995(0.94,1.04)$, and $1.51(1.41,1.61)$, respectively (Figure 2B). We could not analyze lower-income countries and lower-middle-income countries during peak and non-peak mortality periods due to missing information.

\section{Discussion}

Our paper analyses the capacity of healthcare resources worldwide and assesses how this capacity is associated with COVID-19 mortality during peak and non-peak mortality periods of the pandemic. This approach provides insights into system capacity during peak mortality periods with important implications for public health authorities. Despite overall better healthcare resources in High-income countries, these countries had the highest COVID-19 deaths per population. Worldwide, a significantly lower COVID-19 MRR was observed in association with access to more hospital beds. However, we noticed a difference in the pattern of this association, during peak and non-peak mortality periods in middle-income countries (a declining trend in both peak and non-peak mortality periods) and High-income countries (where MRR was $>1$ during peak period even with the highest number of hospital beds).

The COVID-19 pandemic has burdened healthcare systems, and the related consequences are not entirely clear yet. One of the biggest questions for public and health authorities is regarding the extent to which redesign of healthcare systems may change COVID-19 mortality. In an ecological study of 185 countries, High-income countries had a significantly higher number of intensive care unit beds. ${ }^{[8]}$ In this study, COVID-19 mortality had a weakly positive association with the number of intensive care unit beds and a non-significant association with hospital and acute care beds. ${ }^{[8]}$ However, since the authors used linear regression analysis for data with non-normal distributive patterns, these results should be considered with caution. In another study of 86 countries, as of 30th April 2020, countries with greater healthcare capacities had fewer deaths per case during the pandemic. ${ }^{[9]}$ Likewise, our analyses indicate that access to a greater number of hospital beds reduced COVID-19 MRR significantly. Additionally, in this study, we have 
compared COVID-19 peak and non-peak mortality periods to provide a more in-depth analysis of healthcare capacity during the crisis. ${ }^{[9]}$

Despite overall better healthcare resources in High-income countries (Supplemental Figure 2), these countries had the highest COVID-19 deaths per population. This finding may be explained by High-income countries having older populations with underlying chronic conditions as we have previously shown. ${ }^{[1,2]}$ Additionally, the highest percentages of people aged $\geq 65$ years were seen in High-income countries, followed by upper-middle-income countries. A combination of ageing, frailty, and comorbidities can put these individuals at higher risk for COVID-19 mortality. Older adults also have slower and less efficient immune responses than younger adults, making them more vulnerable to complications of COVID-19. ${ }^{[10]}$ In addition, a substantial number of COVID-19 cases and deaths occur among residents in residential aged care facilities and assisted living facilities, where individuals often have preexisting chronic conditions. ${ }^{[11,12]}$ It should be noted that we cannot rule out the possibility of under-reporting COVID19 cases due to a reduced capacity for testing and active surveillance in Lower-middle-income countries.

The pattern of COVID-19 mortality rate differed during peak mortality periods in Middle-income countries vs. Highincome countries. In Middle-income countries, including both Lower-Middle-income countries and Upper-Middleincome countries, we observed a lower COVID-19 mortality rate with a higher number of beds, in both peak and nonpeak mortality periods. In Upper-Middle-income countries during peak mortality periods specifically, there was a nonsignificant increase in COVID-19 mortality rate even in the presence of a higher number of hospital beds. Despite a declining association between the number of hospital beds and COVID-19 mortality rate during peak mortality periods in High-income countries, COVID-19 MRR remained high even with the highest percentile of hospital beds per population (Figure 2-C). We do not have an adequate explanation for these findings in Upper-Middle-income countries and High-income countries. It seems that the exponentially increased number of deaths during peak mortality periods were beyond the direct effect of new confirmed COVID-19 cases. After controlling the effects of other variables such as healthcare resources, income, NCD DALYs, and ageing, the death-to-case ratio increased by approximately two times during the peak periods compared to non-peak mortality periods worldwide (Table 2). Therefore, there is a possibility of overwhelming healthcare systems during peak mortality periods. In the majority of countries, particularly in High-income countries, the death-to-case ratio increased significantly during peak mortality periods (Supplemental Figure 3). In low-income countries, the greatest increase in death-to-case ratio during peak compared to non-peak mortality periods occurred in Yemen, Niger, and Chad. We also observed a significant change in the death-to-case ratio clusters during peak mortality periods (Supplemental Figure 4). These are important findings for public health authorities, who should adjust national healthcare programs to allow adaptable responses to the dynamic phases of the pandemic.

In addition, we observed a significant rise in COVID-19 mortality rate due to the burden of NCDs during COVID-19 peak mortality periods. The burden of NCDs, measured by DALYs, increased deaths by about 2.8 times during peak and 4.2 times during non-peak mortality periods in High-income countries (Figure 2). The course and impact of COVID-19 is influenced by NCDs and possible social inequities present in our societies. ${ }^{[13]}$ COVID-19 is "not a pandemic"; it is "a syndemic" with multiple interactions between communicable and non-communicable diseases. ${ }^{13}$ Due to social isolation, lack of adequate follow-up, and limited healthcare resources, we may observe worsening of pre-existing non-communicable diseases in COVID-19 survivors. ${ }^{[14]}$ The pandemic has burdened healthcare systems and impacted the effectiveness of preventive measures for NCDS, which may have a direct and indirect effect on COVID19 mortality. While we are waiting for widespread public vaccination, an adequate plan to control COVID-19 spread should especially protect people with pre-existing NCDs. Otherwise, we may expect higher mortality and morbidity worldwide. 
Control of the COVID-19 syndemic requires a thorough public health program that considers the interaction of NCDs with COVID-19, especially in High-income countries. Disparities in care for older people with pre-existing chronic diseases in High-income countries vs. Lower-middle-income countries may have contributed to the significant rise of COVID-19 mortality due to NCD-DALYs during non-peak and, particularly, peak mortality periods (Figure 2). In Lowermiddle-income countries, most people living with chronic diseases can rely on care provided by family members or neighbours. ${ }^{[15]}$ The majority of this care in Lower-middle-income countries is provided by women, who account for three-quarters of all time spent on paid and unpaid care responsibilities at home. ${ }^{[15]}$ Because these people are at home, there is a decreased risk of COVID-19 for caregivers and the elderly with pre-existing chronic diseases. In addition, in Lower-middle-income countries, only a small number of older citizens who can afford paid care can enter long-term care facilities and assisted living homes. In contrast, in High-income countries (where long-term care facilities are more available), several COVID-19 cases and deaths occur among residents of these facilities. ${ }^{[16-18]}$ Improving the quality of surveillance in the long-term care population is a priority for ensuring this population's safety from COVID-19. Although there is a more significant association between NCD-DALYs and COVID-19 mortality in Highincome countries, the combination of COVID-19 with NCDs is a global threat. It indeed may affect Lower-middleincome countries with limited resources in primary healthcare prevention. ${ }^{[19,20]}$ The greatest increases in death-tocase ratios during peak mortality periods happened in Yemen, Niger, and Chad.

The COVID-19 syndemic is learning that it is necessary to build global health services into holistic publicly financed universal health care systems that require investment in the foundations of health for all with integrated core capacities for public health and aligned across all levels of governance. ${ }^{[21]}$

Our study has some limitations. Since this is an ecological study, it is susceptible to the ecological fallacy. ${ }^{\text {[22] }}$ Therefore, factors associated with COVID-19 mortality at the national level may not necessarily correlate at the individual level in the same manner. ${ }^{[23]}$ Likewise, to avoid any problematic interpretations, readers need to be cautious of making causal inferences because the results of this study indicate correlations and not causations.

We did not include the number of COVID-19 tests in our analytic models due to missing information in a substantial number of countries. There are also differences in COVID-19 testing policies between countries, affecting the number of tests and confirmed cases globally. ${ }^{[24,25]}$ Similarly, due to missing data in Lower-income countries, we only compared data between and within the Middle-income countries and High-income countries. Finally, a highly dynamic pattern of COVID-19 spread across the globe, and possible unstudied confounders may change our current findings. The major strength of our study is using multiple verified databases and extended statistical models to provide accurate information about the interrelationship of healthcare systems and COVID-19 mortality worldwide. To the best of our knowledge, this is the first ecological study of 139 countries that provides supportive information regarding the idea that COVID-19 is a syndemic.

\section{Conclusion}

Our study emphasizes the importance of efficient hospital care in the prevention of COVID-19 deaths. A triad of ageing, NCDs, and COVID-19 mortality can result in higher pressure on healthcare systems and may overwhelm them. During this critical stage of the pandemic, it is crucial to allocate resources to protect vulnerable populations and avoid further depletion of global healthcare resources. Despite the importance of social isolation during local and national lockdowns, health authorities can reduce additive deaths by implementing an effective plan to manage noncommunicable diseases. COVID-19 is a syndemic but is indeed a preventable condition. 


\section{Abbreviations}

Non-communicable diseases (NCDs)

Disability-adjusted life years (DALYs)

Burden of non-communicable diseases (NCD-DALYs)

Centre for System Science and Engineering (CSSE)

Global burden of disease study (GBD)

World Health Organization (WHO)

Gross domestic product (GDP)

Mortality rate ratio (MRR)

Access to healthcare (HAQI)

\section{Declarations}

Ethics approval and consent to participate. Not applicable

Consent for publication. Not applicable

Availability of data and materials. The dataset generated and analyzed during the current study are available in references [3:7] and in a supplementary table 1-4.

Financial support. This research did not receive any specific grant from funding agencies in the public, commercial, or not-for-profit sector.

Competing interests. The authors declare no conflicts of interest to disclose.

\section{Authors' contributions}

Study concept and design: Mario Di Napoli, M. Reza Azarpazhooh, Farid Najafi, Jose Biller. Data extraction and acquisition of data: Masoud Tokazebani Belasi, Sepideh Kazemi Neya, Mitra Darbandi, Humayon Akhuanzada, Malik Zain. Data analysis and interpretation of data: Shahram Arsang-Jang, Mitra Darbandi Preparation of the first draft of the manuscript: Mario Di Napoli, M. Reza Azarpazhooh, Shahram Arsang-Jang, Masoud Tokazebani Belasi, Farid Najafi, Mitra Darbandi, Malik Zain, Humayon Akhuanzada, Nawaf Yassi, Jose Biller, Ramin Zand, Sepideh Kazemi Neya, Negar Morovatdar, Saverio Stranges. Preparation of the Figures and Tables: Shahram Arsang-Jang, Masoud Tokazebani Belasi, Humayon Akhuanzada, Mitra Darbandi, M. Reza Azarpazhooh. S Arsang-Jang and M Tokazebani Belasi are joint first authors

All authors critically revised the manuscript for relevant intellectual content.

Acknowledgements. Not applicable 


\section{References}

1. Azarpazhooh MR, Amiri A, Morovatdar N, Steinwender S, Rezaei Ardani A, Yassi N, et al. Correlations between COVID-19 and burden of dementia: An ecological study and review of literature. J Neurol Sci. 2020 Jul 4;416: 117013.

2. Azarpazhooh MR, Morovatdar N, Avan A, Phan TG, Divani AA, Yassi N, et al. COVID-19 Pandemic and Burden of Non-Communicable Diseases: An Ecological Study on Data of 185 Countries. J Stroke Cerebrovasc Dis. 2020 Sep;29(9):105089.

3. Roser M, Ritchie H, Ortiz-Ospina E, Hasell J. Coronavirus Pandemic (COVID-19). Our World in Data [Internet]. 2020 [cited 2020 Sep 16]; Available from: https://ourworldindata.org/coronavirus.

4. GBD 2019 Diseases and Injuries Collaborators. Global burden of 369 diseases and injuries in 204 countries and territories, 1990-2019: a systematic analysis for the Global Burden of Disease Study 2019. Lancet. 2020 Oct 17;396(10258):1204-22.

5. GBD 2016 Healthcare Access and Quality Collaborators. Measuring performance on the Healthcare Access and Quality Index for 195 countries and territories and selected subnational locations: a systematic analysis from the Global Burden of Disease Study 2016. Lancet. 2018 Jun 2;391(10136):2236-71.

6. WHO | WHO Global Health Workforce Statistics [Internet]. [cited 2020 Dec 13]. Available from: https://www.who.int/hrh/statistics/hwfstats/en/

7. World Bank Country and Lending Groups - World Bank Data Help Desk [Internet]. [cited 2020 Sep 15]. Available from: https://datahelpdesk.worldbank.org/knowledgebase/articles/906519-world-bank-country-and-lendinggroups

8. Sen-Crowe B, Sutherland M, McKenney M, Elkbuli A. A Closer Look Into Global Hospital Beds Capacity and Resource Shortages During the COVID-19 Pandemic. J Surg Res. 2020 Nov 24; 260: 56-63.

9. Khan JR, Awan N, Islam MM, Muurlink O. Healthcare Capacity, Health Expenditure, and Civil Society as Predictors of COVID-19 Case Fatalities: A Global Analysis. Front Public Health. 2020 Jul 3; 8: 347.

10. Nikolich-Zugich J, Knox KS, Rios CT, Natt B, Bhattacharya D, Fain MJ. SARS-CoV-2 and COVID-19 in older adults: what we may expect regarding pathogenesis, immune responses, and outcomes. Geroscience. 2020 Apr 10; 42(2): 505-14.

11. ECDC Public Health Emergency Team, Danis K, Fonteneau L, Georges S, Daniau C, Bernard-Stoecklin S, et al. High impact of COVID-19 in long-term care facilities, suggestion for monitoring in the EU/EEA, May 2020. Euro Surveill. 2020; 25(22).

12. Zimmerman S, Sloane PD, Reed D. Dementia prevalence and care in assisted living. Health Aff (Millwood). 2014 Apr; 33(4): 658-66.

13. Horton R. Offline: COVID-19 is not a pandemic. Lancet. 2020 Sep 26; 396(10255): 874.

14. Palmer K, Monaco A, Kivipelto M, Onder G, Maggi S, Michel J-P, et al. The potential long-term impact of the COVID-19 outbreak on patients with non-communicable diseases in Europe: consequences for healthy ageing. Aging Clin Exp Res. 2020 Jul; 32(7): 1189-94.

15. Alzheimer's Disease Internationa. World Alzheimer Report 2019 [Internet]. y Alzheimer's Disease International (ADI), London; 2019 [cited 2020 Dec 26]. Available from: https://www.alzint.org/u/WorldAlzheimerReport2019.pdf

16. Arons MM, Hatfield KM, Reddy SC, Kimball A, James A, Jacobs JR, et al. Presymptomatic SARS-CoV-2 Infections and Transmission in a Skilled Nursing Facility. N Engl J Med. 2020 May 28; 382(22): 2081-90.

Page $10 / 15$ 
17. Kimball A, Hatfield KM, Arons M, James A, Taylor J, Spicer K, et al. Asymptomatic and Presymptomatic SARSCoV-2 Infections in Residents of a Long-Term Care Skilled Nursing Facility - King County, Washington, March 2020. MMWR Morb Mortal Wkly Rep. 2020 Apr 3; 69(13): 377-81.

18. D'Adamo H, Yoshikawa T, Ouslander JG. Coronavirus Disease 2019 in Geriatrics and Long-Term Care: The ABCDs of COVID-19. J Am Geriatr Soc. 2020 Apr 16; 68(5): 912-7.

19. Kraef C, Juma P, Kallestrup P, Mucumbitsi J, Ramaiya K, Yonga G. The COVID-19 Pandemic and NonCommunicable Diseases-A Wake-up Call for Primary Health Care System Strengthening in Sub-Saharan Africa. J Prim Care Community Health. 2020 Dec; 11: 2150132720946948.

20. Hernández-Galdamez DR, González-Block MÁ, Romo-Dueñas DK, Lima-Morales R, Hernández-Vicente IA, Lumbreras-Guzmán M, et al. Increased Risk of Hospitalization and Death in Patients with COVID-19 and Preexisting Noncommunicable Diseases and Modifiable Risk Factors in Mexico. Arch Med Res. 2020 Jul 22; 51 (7): 683-9.

21. Lal A, Erondu NA, Heymann DL, Gitahi G, Yates R. Fragmented health systems in COVID-19: rectifying the misalignment between global health security and universal health coverage. Lancet. 2021 Jan 2; 397 (10268): 61-7.

22. Wakefield J. Ecologic studies revisited. Annu Rev Public Health. 2008; 29: 75-90.

23. Pearce N. The ecological fallacy strikes back. J Epidemiol Community Health. 2000 May; 54(5): 326-7.

24. Shaman J. An estimation of undetected COVID cases in France. Nature. 2020 Dec 21.

25. Li R, Pei S, Chen B, Song Y, Zhang T, Yang W, et al. Substantial undocumented infection facilitates the rapid dissemination of novel coronavirus (SARS-CoV-2). Science. 2020 May 1; 368(6490): 489-93.

\section{Tables}

Table 1: The association between COVID-19 mortality and hospital beds: Results of the multilevel generalized model analysis among 139 countries. 


\begin{tabular}{|c|c|c|c|c|c|}
\hline & MRR & SE & $\mathrm{z}$ & P-value & $95 \% \mathrm{Cl}$ for MRR \\
\hline \multicolumn{6}{|l|}{ Variables } \\
\hline \multicolumn{6}{|c|}{ Hospital beds per population* } \\
\hline $40^{\text {th }}$ to $<60^{\text {th }}$ percentile & $0 \cdot 705$ & 0.019 & -12.12 & $<0.0001$ & [0.67 to 0.75$]$ \\
\hline $60^{\text {th }}$ to $<80^{\text {th }}$ percentile & 0.536 & 0.018 & -17.20 & $<0.0001$ & [0.54 to 0.61$]$ \\
\hline$>80^{\text {th }}$ percentile & 0.489 & $0 \cdot 019$ & -17.57 & $<0.0001$ & [0.49 to 0.56$]$ \\
\hline Out-of-pocket payments & 0.984 & 0.0001 & -29.1 & $<0.0001$ & [0.99 to 0.99$]$ \\
\hline \multicolumn{6}{|l|}{ Population density ** } \\
\hline $1^{\text {st }}$ to $2^{\text {nd }}$ quartile & 1.45 & 0.03 & 16.98 & $<0.0001$ & [1.38 to 1.51$]$ \\
\hline $2^{\text {nd }}$ to $3^{\text {rd }}$ quartile & 1.08 & 0.02 & 3.73 & $<0.0001$ & [1.04 to 1.13$]$ \\
\hline$>3^{\text {rd }}$ quartile & 0.804 & 0.019 & -9.12 & $<0.0001$ & [0.76 to 0.84$]$ \\
\hline First component $\S$ & 1.8 & 0.007 & 54.25 & $<0.0001$ & [1.76 to 1.84$]$ \\
\hline Second component $\S$ & 0.929 & 0.013 & -1.4 & $<0.0001$ & [0.81 to 0.83$]$ \\
\hline \multicolumn{6}{|c|}{$\begin{array}{l}\text { Abbreviations: MRR: Mortality rate ratio; SE: Standard error; CI: Confidence interval } \\
\text { * Reference: Hospital bed per } 10,000 \text { population }<40^{\text {th }} \text { percentile; } * \text { References: } 1^{\text {st }} \text { quartile } \\
\text { § Principal components of collinear variables include: Non-communicable disease disability-adjusted life years, } \\
\text { health access and quality index, health-adjusted life expectancy, gross domestic product per capita, and median } \\
\text { age. }\end{array}$} \\
\hline
\end{tabular}

Table 2: COVID-19 death-to-cases during non-peak and peak mortality periods: Total case and death number as of March 23, 2021, and results of Poisson-Tweedie mixed-effects model. 


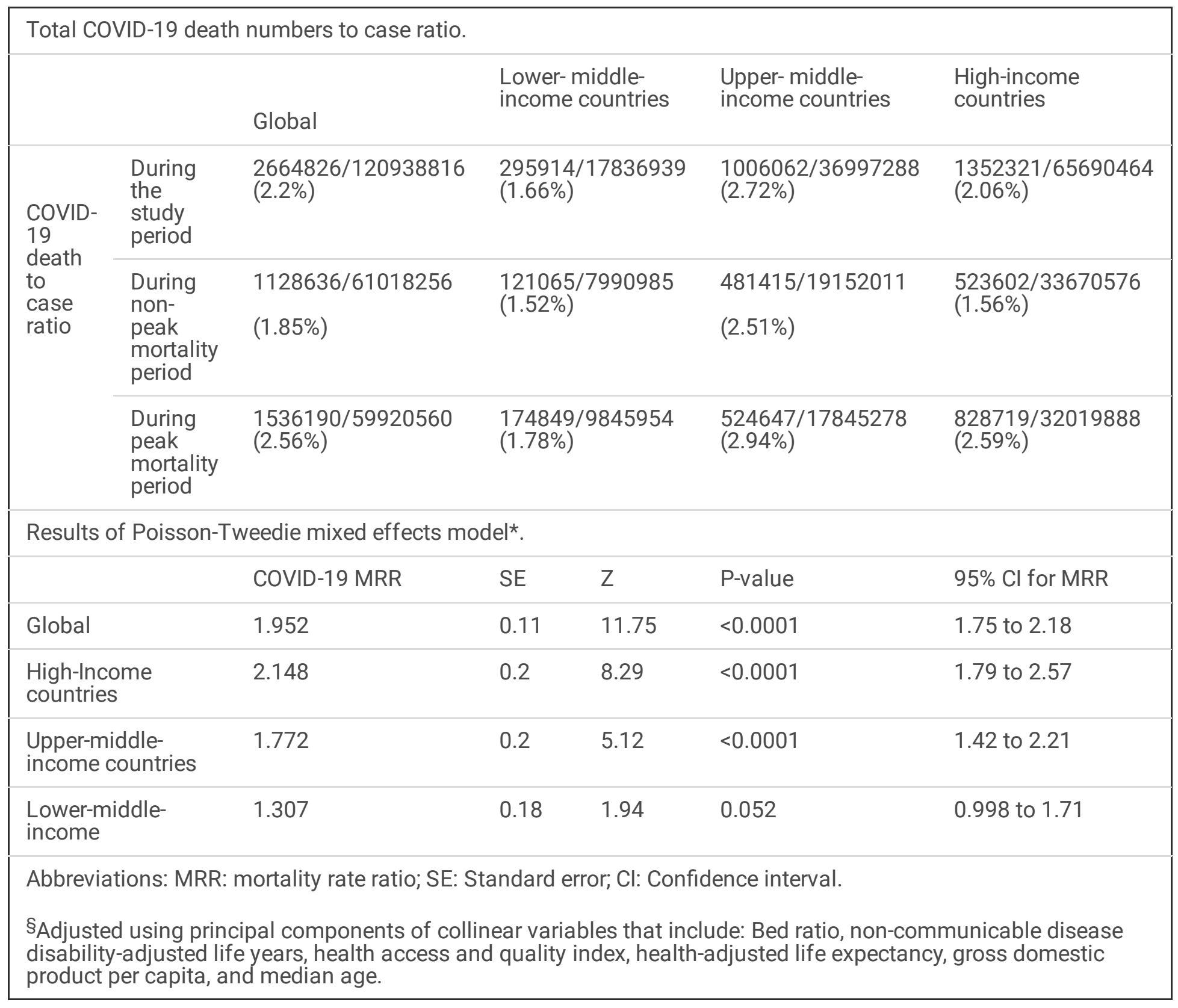

\section{Figures}




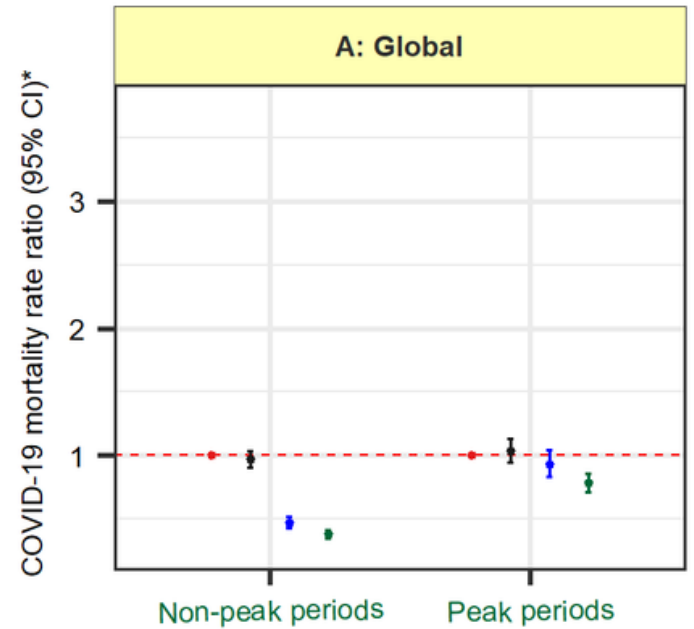

Non-peak periods Peak periods

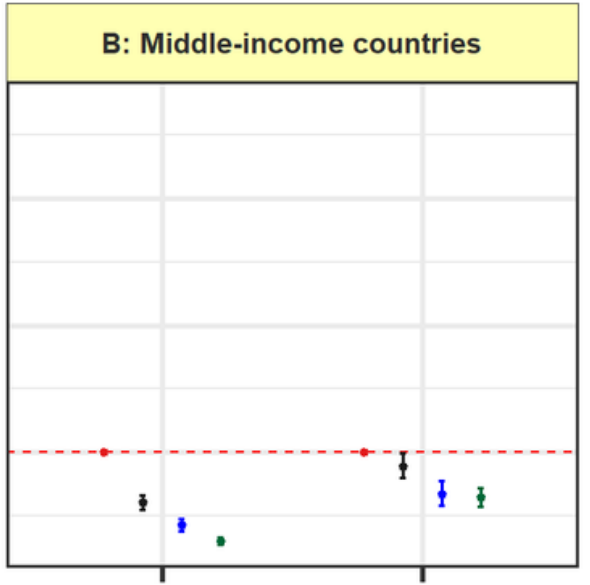

Non-peak periods Peak periods

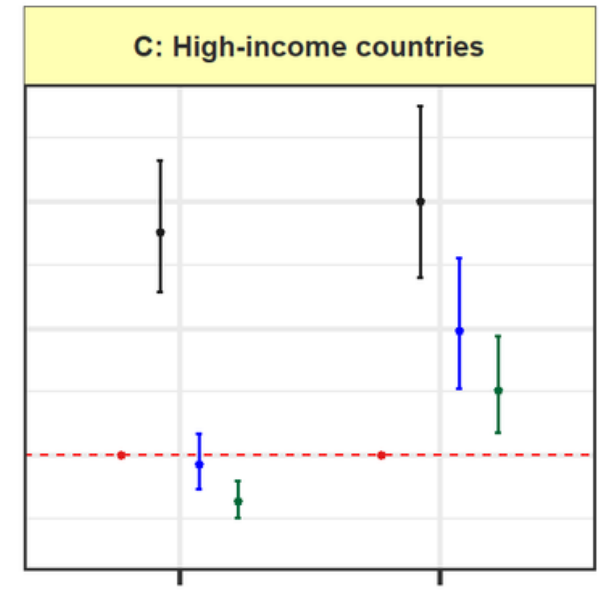

Non-peak periods Peak periods

COVID-19 mortality periods

Hospital beds $\rightarrow<20$ th percentile (reference) $\rightarrow-20$ th to $<40$ th percentile $\rightarrow$ 40th to $<60$ th percentile $\rightarrow>60$ th percentile

Error bars above or below the dashed line indicate statistically significant increase or decrease in COVID-19 mortality rate due to the hospital bed ratio. *Reference category: Hospital bed ratio $<20$ th percentile

Figure 1

(A-C): The effect of hospital beds on COVID-19 mortality rate: A worldwide comparison between COVID-19 peak and non-peak mortality periods.
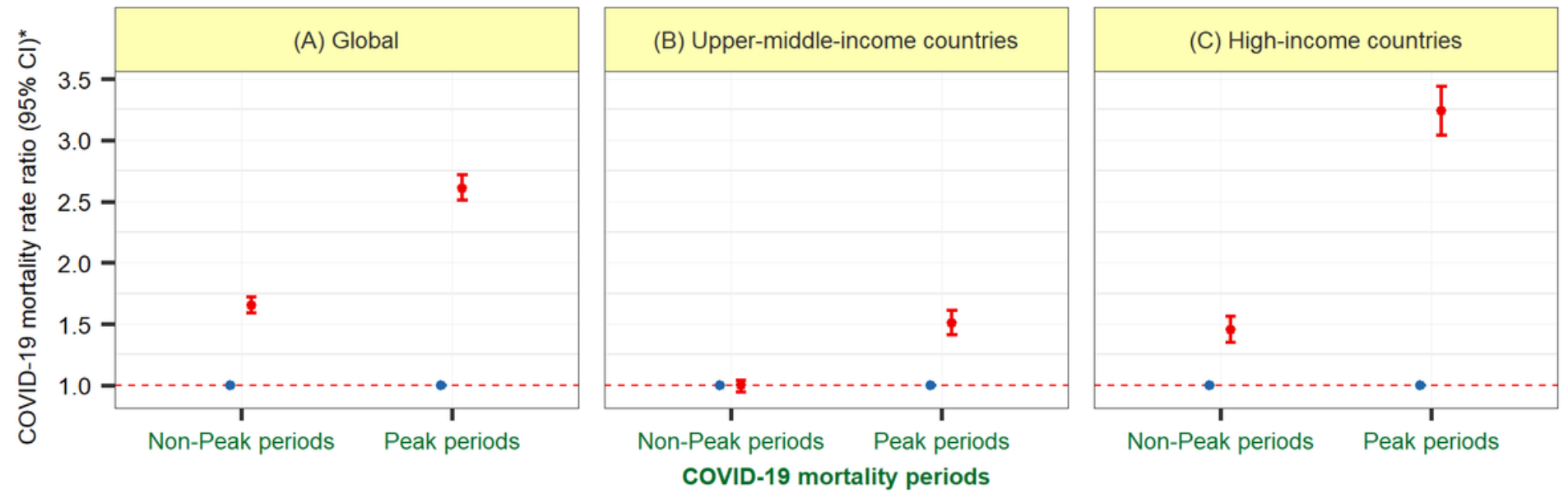

* NCD DALYs < percentile 50th (reference) $*$ NCD DALYs $\geq$ percentile 50th

\section{Figure 2}

(A-C): The association between the burden of non-communicable diseases and COVID-19 mortality rate ratio.

\section{Supplementary Files}

This is a list of supplementary files associated with this preprint. Click to download. 
- SupplementalTable1.docx

- SupplementalTable2.docx

- SupplementalTable3.docx

- SupplementalTable4.docx

- SupplementalFigure1.png

- SupplementalFigure2.png

- SupplementalFig3.png

- SupplementalFig4AC.png 